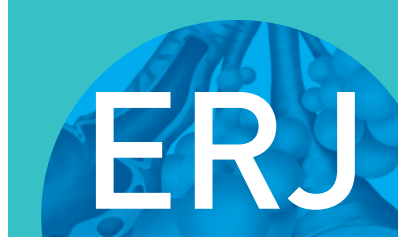

open research
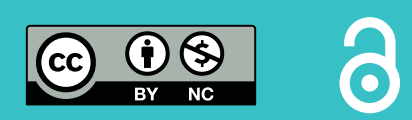

\section{Are respiratory specialist registrars trained to teach?}

\section{To the Editor:}

Imparting medical knowledge to undergraduate and graduate learners in respiratory medicine is a multifaceted process. Consultants deliver part of this teaching but registrars also have many teaching interactions with the medical team, both in formal settings and in day-to-day clinical practice.

The role of respiratory specialist registrars (SpRs) as educators in the clinical setting has not been reviewed in detail in the past. In our previous work, we developed and used a survey tool that was shown to have acceptable psychometric properties [1]. In the single-centre US experience, SpRs/fellows on the Harvard Pulmonary and Critical Care (HPCC) programme were questioned about the training they received in teaching skills, and how this impacted their ability and confidence as teachers, as well as their plans for further medical education roles in their careers. They proved interested in improving their teaching skills but were unclear on the steps they needed to take to actually achieve improvement.

Given the lack of data regarding international perspectives on medical education skills and career significance, in this study, we applied our validated survey to the Irish national respiratory medicine higher specialist training scheme. Our aim was to determine the frequency with which respiratory SpRs were observed when teaching and if they received feedback on their teaching. We planned to address the meaningfulness of this feedback and the effect of training on SpRs' self-reported confidence in their teaching abilities. Underpinning these questions, we wished to determine the SpRs' interest in medical education as a component of their future careers.

We assessed the experience of whether and how teaching skills are imparted to the respiratory SpRs using an online survey (developed in and delivered by Surveymonkey (Palo Alto, CA, USA)). The study protocol was reviewed by programme directors of the Irish respiratory SpR programmes and the protocol was exempted from formal ethical review.

The survey was distributed anonymously through the national training body for postgraduate medical training, the Royal College of Physicians of Ireland. The survey was sent on three occasions to maximise response rate.

The survey addressed many areas of training in teaching techniques, including the frequency of direct observation of SpRs' teaching by a consultant, frequency and perceived quality of feedback from consultants about their teaching, importance and comfort levels with their teaching abilities, and the importance of medical education as a component of their future careers. The survey was adapted from the HPCC study to account for differences in terminology and structure of the Irish training [1]. Results were analysed with GraphPad Prism 4 (GraphPad Software, San Diego, CA, USA).

The response rate to the questionnaire was $68 \%$ (30 out of a possible 44 ). The majority of Irish SpRs were infrequently observed teaching medical students or junior colleagues, with $76 \%$ of Irish SpRs reporting frequency as either "infrequently" or "never" (figure 1a). 57\% of Irish SpRs "rarely" or "never" received feedback from a consultant about their teaching (figure 1b). When asked how often they receive feedback that they felt was useful, 57\% answered "rarely" or "never" receiving useful feedback. For those who did receive feedback, all but one individual felt that they rarely, if ever, disagreed with the feedback they received (i.e. 92\%).

When asked if it was clear what skills were necessary to improve their teaching abilities, $7 \%$ stated that it was "extremely clear", 30\% "clear", 13\% "moderately clear", 33\% "somewhat clear" and 17\% "not at all clear". In this setting, $85 \%$ of Irish SpRs were either interested or extremely interested in dedicated training in order to acquire these skills. Over time, SpRs felt that their comfort level with teaching improved (figure 1c); 63\% felt "more comfortable" since commencing specialist training, although 10\% actually felt "less comfortable".

When questioned on the importance of teaching for their future careers, 96\% of SpRs indicated that teaching is "very important" or "important" (figure 1d). Overall, $81 \%$ of SpRs were interested in pursuing a career as a medical educator. 
a)

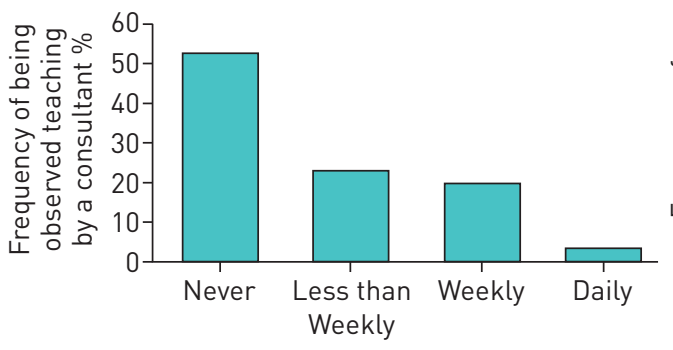

c)

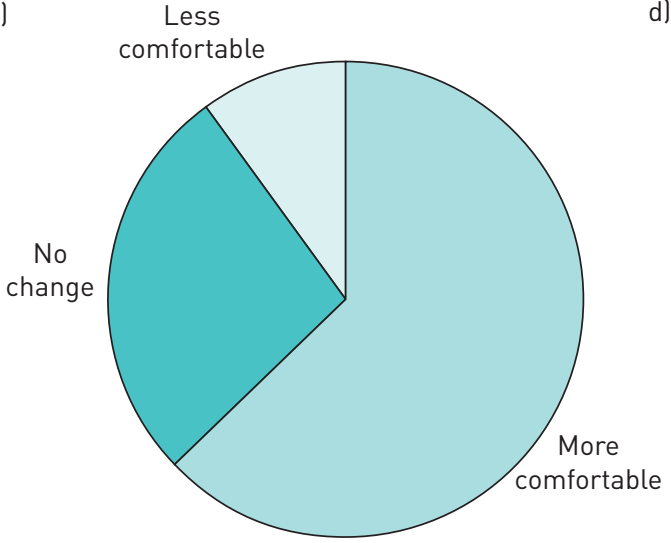

b)

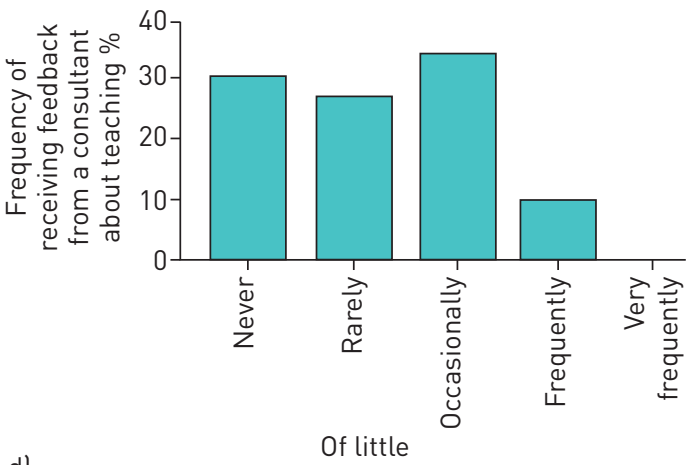

FIGURE 1 a) Frequency of observation: when asked about the frequency of being observed teaching by an attending, 76\% of respondents responded "less than weekly" or "never". b) Frequency of receiving feedback: $57 \%$ of Irish specialist registrars (SpRs) "rarely" or "never" received feedback from a consultant about their teaching. c) Comfort levels with teaching: when asked about their comfort teaching since commencing specialist training, only $63 \%$ of Irish SpRs felt "more comfortable" and $10 \%$ of the respondents actually felt "less comfortable". d) Importance of teaching skills to future career: 97\% of Irish SpRs considered teaching skills to be "very important" or "important" to their future careers. Only 3\% of the Irish cohort considered teaching skills to be of "minimal" or "moderate" importance.

In this study, SpRs training in respiratory medicine in Ireland were very interested in developing their skills and abilities as medical educators. It was rare for them to be formally observed when teaching and rarer still for them to receive feedback regarding their teaching abilities.

A very positive finding in this study, and a promising observation for the future, is the strong interest that the respiratory SpRs have in developing more in-depth knowledge of medical education and teaching skills as part of their future careers. The results serve as needs assessment to guide improvements in how SpRs are trained to be educators. Potential options to address this need would include "teaching fellowships" [2-5], although the incorporation of teaching skills within a respiratory training programme might best suit the clinician-educator track model [6]. The interest in a career as a medical educator in the Irish respiratory SpRs was high and at a similar level to that found in the USA, indicating potential receptivity to such a training pathway within respiratory specialist training programmes in both Ireland and the USA.

There are many ways to impart knowledge of teaching skills, including formal courses, lectures and small group sessions involving role play. Observation of teaching and providing feedback on this teaching is only one part of developing better teaching skills. However, an advantage of direct observation and feedback is that compared to other techniques, direct observation and feedback is individualised and provides focussed information to the trainee, intended to directly benefit the trainee's teaching skills [7].

Feedback itself is a cornerstone of medical training. 62\% percent of SpRs had either "rarely" or "never" received feedback regarding their teaching skills, compared to only $30 \%$ of their US counterparts [1]. In the competency-based approach to medical education, feedback is a key component, facilitating opportunities to improve and encouraging trainees to reach their maximum potential $[6,8]$. Feedback after direct observation should also be a key element to guide fellows as they improve their teaching skills. The provision of meaningful feedback is a skill that can be taught [9]. It is interesting to note that trainees can 
report that feedback is given infrequently and/or ineffectively $[10,11]$, whereas instructors may feel that they provide frequent and adequate feedback [11].

Confidence in teaching ability is influenced by many factors, and a direct association with the lack of observation and feedback has not been proven here. The US fellows reported higher comfort levels as a teacher than the Irish fellows [1]. A recently published study reviewed the experience of paediatric fellows involved in delivering simulation model education sessions to residents and medical students, and found that self-rated confidence improved immediately after participation but returned to baseline 6 months later [12].

In conclusion, Irish respiratory SpRs are enthusiastic to be involved in teaching and are eager to develop their teaching skills. They are only occasionally observed when teaching, and are generally receptive to feedback on their teaching but do not receive enough of it. Investing in training SpRs to develop better teaching skills is likely to be beneficial and valued, as most see medical education as a major part of their future careers.

Emer Kelly ${ }^{1}$, Sinead M. Walsh ${ }^{1}$ and Jeremy B. Richards ${ }^{2}$

${ }^{1}$ Department of Respiratory Medicine, St Vincent's University Hospital, University College Dublin and Royal College of Physicians of Ireland, Dublin, Ireland. ${ }^{2}$ Beth Israel and Deaconess Medical Center, and Harvard Medical School, Boston, MA, USA.

Correspondence: Emer Kelly, St Vincent's University Hospital, Elm Park, Dublin 4, Ireland.

E-mail: emer.kelly@svuh.ie

Received: April 292015 | Accepted: June 082015

Conflict of interest: None declared.

@ERSpublications

Respiratory SpRs are interested in medical education but rarely observed when teaching and receive little feedback http://ow.ly/Our0m

Copyright $\odot$ ERS 2015. This article is open access and distributed under the terms of the Creative Commons Attribution Non-Commercial Licence 4.0 .

Acknowledgments: The authors would like to acknowledge the assistance of Terence O'Connor and Richard Costello (Royal College of Physicians of Ireland, Dublin, Ireland). We would also like to thank the fellows and specialist registrars for taking the time to complete the surveys in this study.

\section{References}

1 Richards JB, Kelly E, Fessler H, et al. A novel survey tool to assess pulmonary and critical care fellows' attitudes regarding acquiring teaching skills during fellowship training. J Grad Med Educ 2013; 5: 506-509.

2 Qureshi S. Teaching fellowships for UK foundation doctors. Med Teach 2014; 1-2.

3 Muller JH, Irby DM. Developing educational leaders: the teaching scholars program at the University of California, San Francisco, School of Medicine. Acad Med 2006; 81: 959-964.

4 Steinert Y, Boudreau JD, Boillat M, et al. The Osler Fellowship: an apprenticeship for medical educators. Acad Med 2010; 85: 1242-1249.

5 Robins L, Ambrozy D, Pinsky LE. Promoting academic excellence through leadership development at the University of Washington: the Teaching Scholars Program. Acad Med 2006; 81: 979-983.

6 Krackov SK. Expanding the horizon for feedback. Med Teach 2011; 33: 873-874.

7 Newman LR, Brodsky DD, Roberts DH, et al. Developing expert-derived rating standards for the peer assessment of lectures. Acad Med 2012; 87: 356-363.

8 Krackov SK, Pohl H. Building expertise using the deliberate practice curriculum-planning model. Med Teach 2011; 33: $570-575$

9 Ramani S, Krackov SK. Twelve tips for giving feedback effectively in the clinical environment. Med Teach 2012; 34: 787-791.

10 Hewson MG, Little ML. Giving feedback in medical education: verification of recommended techniques. $J$ Gen Intern Med 1998; 13: 111-116.

11 Branch WT, Jr, Paranjape A. Feedback and reflection: teaching methods for clinical settings. Acad Med 2002; 77: $1185-1188$.

12 Tofil NM, Peterson DT, Harrington KF, et al. A novel iterative-learner simulation model: fellows as teachers. J Grad Med Educ 2014; 6: 127-132. 